RUNNING HEAD: Anomalies to Markowitz's Hypothesis

\title{
Anomalies to Markowitz's Hypothesis and a Prospect-Theoretical Interpretation
}

\author{
Marc Scholten $^{\mathrm{a}}$ and Daniel Read ${ }^{\mathrm{b}}$
}

${ }^{a}$ ISPA University Institute

Rua Jardim do Tabaco 34

1149-041 Lisboa, Portugal

Tel: 00351218811700

Fax: 00351218860954

E-Mail: scholten@ispa.pt

(Corresponding author)

${ }^{b}$ Warwick Business School

Scarman Road

The University of Warwick

Coventry, CV4 7AL, United Kingdom

Tel: 00442476524306

Fax: 00442476523719

Email: Daniel.Read@wbs.ac.uk 


\begin{abstract}
Markowitz hypothesized a fourfold pattern of risk preferences, with risk aversion for large gains and small losses, but risk seeking for small gains and large losses. We test his hypothesis, and obtain two major results. One is the dispersion effect: A majority exhibits risk seeking and risk aversion for small and large gains, but disperses into five preference groups for small and large losses. There are the 'Markowitzians' (risk aversion and risk seeking), the 'non-Markowitzians' (risk seeking and risk aversion), the 'Cautious' (global risk aversion), the 'Audacious' (global risk seeking), and the 'Wavering' (who exhibit no definite preference pattern). The other result is the migration effect: The composition of the preference groups changes across risk levels. More specifically, when going from high to moderate risk levels, the shares of the Markowitzians and the Cautious fall while the shares of the Audacious and the non-Markowitzians rise. We show that, if prospect theory accommodates the dispersion effect by allowing for heterogeneity in the elasticity of the value function and the elevation of the probability-weighing function, it correctly predicts the migration effect.
\end{abstract}

\title{
Keywords
}

Risk aversion, risk seeking, preference heterogeneity, preference uncertainty, Markowitz, prospect theory.

\section{JEL Codes}

C90, C91, D01, D03. 


\section{Anomalies to Markowitz's Hypothesis and a Prospect-Theoretical Interpretation}

Happy families are all alike; every unhappy family is unhappy in its own way (Leo Tolstoy, Anna Karenina).

In most analyses of risky choice, a typical decision maker is assumed to exhibit modal preference patterns. This is true of the earliest analyses (Bernoulli, 1738/1954) and continues through to the present (Brandstätter et al., 2006; Hogarth \& Einhorn, 1990; Kahneman \& Tversky, 1979; Loomes, 2010; Markowitz, 1952). Thus, for instance, if, over a series of choices, modal preferences shift from risk seeking to risk aversion, it is assumed that a typical decision maker exhibits this shift. This typical decision maker is then described by models of individual decision making under risk.

We investigate a series of choices designed by Markowitz (1952). These are choices between a sure thing and a gamble with the same expected value, in a series where the sure thing varies from very small to very large. The preference patterns he observed "informally" motivated the development of what now, with the advent of prospect theory (Kahneman \& Tversky, 1979), would be called a referencedependent theory of risky choice. We conduct a systematic and large-scale investigation of the choice series designed by Markowitz, and find that the predictions of both Markowitz's theory and prospect theory are far off the mark: At the aggregate level (modal preference patterns), the predicted preference patterns are not confirmed, and, at the disaggregate level (individual preference patterns), the typical decision maker does not exist.

We obtain two major results. One is the dispersion effect: In gains, most people exhibit the preference pattern described by Markowitz, but, in losses, they disperse into groups with different preference patterns. The other result is the migration effect: The composition of the preference groups changes across risk levels. We show that, if prospect theory accommodates the dispersion effect by allowing for heterogeneity in the elasticity of the value function and the elevation of the probabilityweighing function, it correctly predicts the migration effect. We discuss implications for the purchase of gambles and insurances, and other issues raised by our investigation. 


\section{Markowitz's Theory:}

\section{A Fourfold Pattern in Outcome Magnitude}

Markowitz (1952) questioned the common assumption that people are globally risk averse, meaning they will prefer a sure thing to a gamble with the same expected monetary value. He reported that respondents in an informal survey were risk averse for large gains and small losses, but risk seeking for small gains or large losses. They preferred a 1/10 chance of gaining $\$ 1$ to gaining 10 cents for sure (risk seeking), but preferred gaining \$1 million for sure to a 1/10 chance of gaining $\$ 10$ million (risk aversion); conversely, they preferred losing 10 cents for sure to a 1/10 chance of losing $\$ 1$ (risk aversion), but preferred a 1/10 chance of losing $\$ 10$ million to losing $\$ 1$ million for sure (risk seeking). To explain this fourfold pattern of risk preferences, which we call the 'M4 pattern,' Markowitz proposed a triply inflected, reference-dependent value function. Like the prospect theory value function, Markowitz's is defined over positive and negative deviations from current wealth (gains and losses). Unlike the prospect theory value function, which is concave in gains and convex in losses, Markowitz's is first convex and only then concave in gains, and first concave and only then convex in losses. Markowitz's theory is an expected value model, meaning that outcome values are weighted by outcome probabilities, and it is for this reason that it needs the triply inflected value function to produce the M4 pattern. As we discuss next, prospect theory can produce the M4 pattern by combining a non-linear probability-weighing function with a special value function.

\section{Prospect Theory:}

\section{The Fourfold Pattern in Outcome Magnitude}

Prospect theory is a non-expected value model. This means that outcome values are weighted by a non-linear transformation of probabilities, so that the value of a gamble is not its expectation, $p v(x)$, as in Markowitz's theory, but the product of a probability weight and the outcome value, $w(p) v(x)$. In prospect theory, the probability-weighing function overweighs low probabilities and underweighs moderate to high ones. If it overweighs Markowitz's 1/10, as is generally assumed, it will contribute to risk seeking in gains and risk aversion in losses. The value function, on the other hand, is concave over gains and convex over 
losses (diminishing sensitivity), contributing to risk aversion in gains and risk seeking in losses. For Markowitzian choices, therefore, the probability-weighing function and the value function work in opposite directions.

The M4 pattern requires that the probability-weighing function outweighs the value function for small outcomes, i.e., $w(1 / 10)>v(x) / v(10 x)$, but is outweighed by it for large ones, i.e., $v(m x) / v(10 m x)>$ $w(1 / 10)$ for some $m>1$. If we combine the two inequalities, we get

$$
\frac{v(m x)}{v(10 m x)}>\frac{v(x)}{v(10 x)} \text { for } m>1
$$

This property of the value function is called decreasing elasticity (al-Nowaihi, \& Dhami, 2009; Scholten \& Read, 2010; see also Loewenstein \& Prelec, 1992): As the magnitude of $x$ increases by constant proportions, the magnitude of $v(x)$ increases by decreasing proportions. For instance, doubling $\$ 100$ yields a smaller proportional increase in value than doubling $\$ 1$. Geometrically, $\log (v(x))$ is a concave function of $\log (x)$.

Decreasing elasticity has not previously been associated with prospect theory. A power value function, as proposed by Tversky and Kahneman (1992), has constant elasticity (within the domains of gains and $\operatorname{losses})$. Geometrically, $\log (v(x))$ is a linear function of $\log (x)$. Both Köbberling and Wakker's (2005) normalized exponential function and Scholten and Read's (2010) normalized logarithmic function are reference-dependent value functions with decreasing elasticity. The Appendix discusses the concept of elasticity more extensively. For gains, the normalized logarithmic function combines decreasing absolute risk aversion with increasing relative risk aversion, the two conditions for utility functions discussed by Abdellaoui et al. (2007), and Holt and Laury (2002), whereas the normalized exponential function combines increasing relative risk aversion with constant absolute risk aversion.

Prospect theory can therefore produce the M4 pattern if it combines an overweighing of low probabilities with a decreasingly elastic value function. However, it can also produce other preference patterns, depending on whether the probability-weighing function outweighs the value function for small 
outcomes, and on how the elasticity in gains compares with the elasticity in losses. We next discuss these predictions.

\section{Prospect Theory:}

\section{A Twofold Pattern and Two Threefold Patterns in Outcome Magnitude}

In this section, we discuss how prospect theory can produce four preference patterns in Markowitzian choices between $x$ and $(10 x, .1)$. We assume that the probability-weighing function is the same for gains and losses. While this assumption may be relaxed (Tversky \& Kahneman, 1992), doing so would not change the predictions we derive from prospect theory; it would only sometimes open different routes to the same predictions. In any event, prospect theory needs decreasing elasticity to produce the M4 pattern, but does not need domain-specific probability weighing to accommodate the evidence we obtain in our experiments. In the remainder of this paper, and unless otherwise indicated, 'prospect theory' will refer to 'prospect theory augmented with decreasing elasticity.' For the choices examined in this paper, between a sure thing $x$ and a gamble $(x / p, p)$, original prospect theory coincides with cumulative prospect theory (Tversky \& Kahneman, 1992).

As just discussed, prospect theory combines diminishing sensitivity to outcomes with the overweighing of low probabilities. Diminishing sensitivity contributes to risk aversion in gains and risk seeking in losses, whereas the overweighing of low probabilities contributes to risk seeking in gains and risk aversion in losses. The predictions of prospect theory depend on the relative strength of these opposing forces. Risk preferences will reverse only if the probability-weighing function outweighs the value function for small outcomes but is outweighed by it for large ones. ${ }^{1}$ That is, when

$$
\frac{v(m x)}{v(10 m x)}>w(1 / 10)>\frac{v(x)}{v(10 x)} \text { for } m>1 .
$$

Risk preferences will never reverse if the probability-weighing function never outweighs the value function. This would yield a twofold pattern of global risk aversion in gains and global risk seeking in losses: 
$\frac{v(m x)}{v(10 m x)}>\frac{v(x)}{v(10 x)}>w(1 / 10)$ for $m>1 .^{2}$

The M4 pattern and the twofold pattern can occur regardless of how elasticity in gains compares with elasticity in losses. There are three logical possibilities: Either elasticity is equal for losses and gains, or it is greater for losses, or it is greater for gains. If elasticity is equal for losses and gains (symmetric elasticity), the value ratio (between the values of the sure thing and the risky outcome) is unchanged by a sign reversal:

$\frac{v(x)}{v(10 x)}=\frac{v(-x)}{v(-10 x)}>\frac{1}{10}$ for $x>0$.

Alternatively, if elasticity is greater for losses (loss amplification; see Prelec \& Loewenstein, 1991), the value ratio is smaller for losses:

$\frac{v(x)}{v(10 x)}>\frac{v(-x)}{v(-10 x)}>\frac{1}{10}$ for $x>0$

Finally, if elasticity is greater for gains (gain amplification), the value ratio is smaller for gains:

$\frac{v(-x)}{v(-10 x)}>\frac{v(x)}{v(10 x)}>\frac{1}{10}$ for $x>0$.

Symmetric elasticity, loss amplification, and gain amplification relate to how close to linearity the value function is in gains and in losses. We can illustrate this with the power value function proposed by Tversky and Kahneman (1992):

$v(x)= \begin{cases}x^{\alpha} & \text { if } x \geq 0 \\ -\lambda(-x)^{\beta} & \text { if } x<0,\end{cases}$

where $0<\alpha, \beta<1$ are diminishing sensitivity (the value function is concave in gains and convex in losses) and $\lambda>1$ is constant loss aversion (see also Tversky \& Kahneman, 1991). Symmetric elasticity means $\alpha=\beta$. Loss amplification means $\beta>\alpha$, and gain amplification means $\alpha>\beta$. The evidence reviewed by Wakker et al. (2007, Note 1) includes cases of both symmetric and asymmetric elasticity, with loss amplification being more prevalent than gain amplification. 
Regardless of whether the elasticity of the value function is symmetric or asymmetric, it is possible that, in both gains and losses, the probability-weighing function outweighs the value function for small outcomes but is outweighed by it for large ones, yielding the M4 pattern, or never outweighs the value function, yielding the twofold pattern. Under symmetric elasticity, and in the absence of domainspecific probability weighing, these are the only two possibilities: The value ratio $v(x) / v(10 x)$ is the same for gains and losses (Expression 1), so that, if $w(1 / 10)$ exceeds the value ratio for small gains, it will exceed the value ratio for small losses as well, or, alternatively, if $w(1 / 10)$ never exceeds the value ratio for gains, it will never exceed the value ratio for losses either. Under asymmetric elasticity, however, two other preference patterns can emerge.

Under loss amplification, the value ratio is smaller for losses than for gains (Inequality 2), so $w(1 / 10)$ can exceed the value ratio for small losses but never exceed the value ratio for gains. This would yield a threefold pattern of global risk aversion in gains, alongside a reversal from risk aversion to risk seeking in losses. Under gain amplification, the value ratio is smaller for gains than for losses (Inequality $3)$, so $w(1 / 10)$ can exceed the value ratio for small gains but never exceed the value ratio for losses. This would yield a threefold pattern of global risk seeking in losses, alongside a reversal from risk seeking to risk aversion in gains.

In sum, for choices between $x$ and $(10 x, .1)$, or '10x choices,' prospect theory produces not only the M4 pattern, but also a twofold pattern and two threefold patterns in outcome magnitude, and thus produces a broader set of predictions than Markowitz's theory does. This situation is unique, however, to choices between a sure thing and a low-probability gamble. We next consider choices between $x$ and ( $2 x$, $.5)$, or ' $2 x$ choices,' for which prospect theory predicts only the twofold pattern in outcome magnitude, while Markowitz's theory continues to predict only the M4 pattern.

\section{Markowitz's Theory and Prospect Theory:}

\section{The Fourfold Pattern and the Twofold Pattern in Outcome Magnitude}

In Markowitz's theory, the value function changes from convex to concave in gains and from concave to convex in losses, so that, for $2 x$ choices as well as $10 x$ choices, the prediction is the M4 pattern. 
In prospect theory, however, $2 x$ choices differ from $10 x$ choices in that the value function and the probability-weighing function both contribute to risk aversion in gains and risk seeking in losses: The value function because of diminishing sensitivity, and the probability-weighing function because it underweighs the moderate probability of .5 . Thus, for $2 x$ choices, prospect theory predicts a twofold pattern of global risk aversion in gains and global risk seeking in losses. This prediction extends to choices between a sure thing and a high-probability gamble, because high probabilities are underweighted as well. We next discuss existing evidence on Markowitz's hypothesis.

\section{Existing Evidence}

The earliest evidence comes from Hershey and Schoemaker (1980, Table 3, rows 13-17). Letting $x$ be the sure outcome and $(x / p, p)$ be a gamble yielding $x / p$ with probability $p$, they set $p$ at .01 , and $x$ at $\$ 1$, $\$ 100, \$ 1,000$, and $\$ 10,000$ (in this section, $x$ stands for the magnitude of $x$, or its absolute value). The results supported Markowitz's hypothesis: As $x$ increased, aggregate choices changed from risk seeking to risk aversion for gains, but from risk aversion to risk seeking for losses. However, choice probabilities were closer to chance level (i.e., closer to a 50/50 distribution) for losses than for gains, a finding that extends beyond Markowitzian choice series (e.g., Schoemaker, 1990). ${ }^{3}$

Hogarth and Einhorn (1990) conducted three studies, in which $p$ was set at .1, .5, and .8 (Study 1, Table 2) or at .1, .5, and .9 (Studies 2 and 3, Table 4), and $x$ was set at $\$ 2, \$ 200$, and $\$ 20,000$ (Study 1, hypothetical payoffs), at $\$ 1$ and $\$ 10,000$ (Study 2, hypothetical payoffs), or at $\$ 0.10$ and $\$ 10$ (Study 3, real payoffs).

In gains, Markowitz's hypothesis was supported at all probability levels: As $x$ increased, aggregate choices moved away from risk seeking and toward risk aversion. For $p=.1$, there was either global but decreasing risk seeking or a reversal from risk seeking to risk aversion; for $p=.5$ and .8 , there was a reversal from risk seeking to risk aversion; for $p=.9$, there was global and increasing risk aversion. The reversals for moderate to high probabilities are inconsistent with prospect theory, because the probabilityweighing function underweighs those probabilities, and thus contributes, together with the concave value function, to risk aversion. 
In losses, the results showed a great diversity. In Study 1, the direction of the results depended on the probability level. For $p=.1$, there was global but decreasing risk aversion, consistent with Markowitz's hypothesis and prospect theory. For $p=.5$, there was a reversal from risk seeking to risk aversion, when Markowitz would predict a reversal in the opposite direction, and prospect theory would predict global and increasing risk seeking, because (1) the probability-weighing function underweighs moderate probabilities, and thus contributes, together with the convex value function, to risk seeking, and (2) the value function is decreasingly elastic. For $p=.8$, there was global but decreasing risk seeking, when Markowitz and prospect theory would predict increasing risk seeking. In Study 2, the results did not depend on the probability level: There was an almost constant level of risk seeking, when Markowitz and prospect theory would predict increasing risk seeking. Finally, in Study 3, the results again depended on the probability level, but were always inconsistent with Markowitz's hypothesis and prospect theory. For $p$ $=.1$, there was a reversal from risk seeking to risk aversion; for $p=.5$, there was global but decreasing risk seeking; for $p=.9$, there was an almost constant level of risk seeking. Across all studies, and as in Hershey and Schoemaker's (1980) study, choice probabilities were closer to chance level for losses than for gains.

The results of the studies reviewed above confirm Markowitz's hypothesis for gains: As outcome magnitude increased, aggregate choices moved away from risk seeking and toward risk aversion. This trend has also been observed in matching (Fehr-Duda et al., 2010) and choice-based matching (e.g., Green et al, 1999). However, the results showed a great diversity for losses. Aggregate choices either moved away from risk aversion and toward risk seeking, as Markowitz hypothesized, or they moved in the opposite direction, or they did not move at all. Moreover, choice probabilities were generally closer to chance level for losses than for gains. The combination of a clear trend in gains and an unclear trend in losses has also been observed in matching (Fehr-Duda et al., 2010). We next offer a possible interpretation of these results. 


\section{An Interpretation of Existing Evidence:}

\section{Dispersion and Migration Effects}

We build an ideal representation of the existing evidence, in which people who exhibit the Markowitzian reversal from risk seeking to risk aversion in gains disperse into five preference groups in losses: (1) The Markowitzians, who exhibit a reversal from risk aversion to risk seeking, (2) the nonMarkowitzians, who exhibit a reversal from risk seeking to risk aversion, (3) The Audacious, who are globally risk seeking; (4) the Cautious, who are globally risk averse, and (5) the Wavering, who do not exhibit a definite preference pattern. This dispersion into different preference groups is the dispersion effect, which involves preference heterogeneity (i.e., different people exhibiting different preference patterns), and, due to the inclusion of the Wavering among the preference groups, preference uncertainty

(i.e., people exhibiting preferences that do not conform to a definite pattern). In our ideal representation of the existing evidence, we will assume that the Wavering have an uncontrollably trembling hand in losses, so that their choices in that domain are completely at random.

The existing evidence includes two results. One is that choice probabilities are closer to chance level for losses than for gains. We see two explanations for this result. One is preference uncertainty: The Wavering bring the aggregate choices closer to a 50/50 distribution. The other explanation is preference heterogeneity: The aggregate choices are brought closer to a 50/50 distribution by the Markowitzians and non-Markowitzians, who reverse their preferences in opposite directions, and by the Audacious and the Cautious, who have globally opposite preferences. Both preference uncertainty and preference heterogeneity contribute to 'risk neutrality' at the aggregate level where such neutrality may not exist at the disaggregate level. Risk neutrality would mean that the value of the sure thing and the value of the gamble are close together, so that people would feel more or less 'indifferent' between the two options (Schneider, 1992). Neither Markowitz's theory nor prospect theory implies a greater indifference in losses than in gains.

The other result, from Hogarth and Einhorn (1990), is that modal preference patterns in losses change across studies and, within studies, across probability levels. In our explanation, this is because the 
composition of the preference groups changes, not because there exists a typical decision maker who exhibits changing preference patterns. The changing composition of the preference groups is the migration effect. We will develop an individual-difference formulation of prospect theory that, by allowing decision makers to disperse into different preference groups, correctly predicts how they will migrate between preference groups across probability levels.

We will report three studies in which investigate preference patterns both at the aggregate and at the disaggregate level. In Experiments 1 and 2, participants make $10 x$ choices and 2x choices, respectively. In both studies, the majority of the participants disperse into the five preference groups identified above (the dispersion effect). Furthermore, the composition of the preference groups changes across $10 x$ and $2 x$ choices (the migration effect). We then develop our individual-difference formulation of prospect theory. However, a comparison between Experiment 1 (10x choices) and Experiment 2 ( $2 x$ choices) does not allow us to directly test how decision makers migrate between preference groups across probability levels (.1 and .5). We therefore conduct Experiment 3, in which the same participants make both $10 x$ and $2 x$ choices. This study confirms our prospect-theoretical interpretation of the results.

\section{Experiments 1 and 2}

Experiments 1 and 2 were conducted independently from one another, but both method and results warrant presenting them together.

\subsection{Method}

\subsubsection{Participants and payment}

The participants were members of the Yale School of Management virtual laboratory (eLab). In Experiment 1, 189 participants included 73 men and 116 women, averaging 35 years of age. In Experiment 2, 255 participants included 92 men and 163 women, averaging 38 years of age. The great majority had college or a Bachelor's degree. Participants were entered in a lottery offering a 1 in 50 chance to win a $\$ 50$ Amazon.com gift certificate. For Markowitzian choice series, incentive compatible payment schedules, even probabilistic ones, are prohibitive. 


\subsubsection{Stimuli and procedure}

In Experiment 1, participants made six $10 x$ choices for gains and for losses. The magnitude of the sure thing ranged from $\$ 0.25$ to $\$ 25,000$ in multiples of 10 , and in that order. In Experiment 2, participants made seven $2 x$ choices for gains and for losses. The magnitude of the sure outcome ranged from $\$ 0.25$ to $\$ 250,000$ in multiples of 10 , and in that order. We fixed the order so as to recreate Markowitz's thought experiment as closely as possible. In Experiment 3, we evaluate whether fixing or randomizing the order has any effect on the results, which it has not. In each experiment, the order of gains and losses was counterbalanced between participants.

On the welcome page, the participants read their rights in participating, including their payment. Once enrolled in the experiment, participants were informed that they would be given two types of choices. Sometimes they would choose between two possible receipts. Other times, they would choose between two possible payments. They were then shown how a choice would look like. They were further informed that, between choices, the screen would be white, and that they would have to hit the "Next question" button to continue. They then proceeded with the experimental trials. An example of a $10 x$ choice in gains, as presented to the participants, is one between "a 1/10 chance of receiving $\$ 250$ " and “definitely receiving $\$ 25 . ”$ An example of a $2 x$ choice in losses is one between "a 1/2 possibility of having to pay $\$ 50$ " and "definitely having to pay $\$ 25$." Upon completing the experimental trials, participants filled in their demographics.

\subsection{Results}

The top panel of Figure 1 shows the aggregate results for the $10 x$ choices. Aggregate choices for gains were monotonically related to their magnitude, and reversed from risk seeking to risk aversion. Aggregate choices for losses were globally risk averse, but were not monotonically related to outcome magnitude. The results for losses contradict both Markowitz's theory, which would predict a reversal from risk aversion to risk seeking, and prospect theory, which would predict either the Markowitzian reversal or global risk seeking. The bottom panel of Figure 1 shows the aggregate results for the $2 x$ choices. Again, aggregate choices for gains were monotonically related to their magnitude, and reversed from risk seeking 
to risk aversion. The results for gains contradict prospect theory, which would predict global risk aversion. Aggregate choices for losses also reversed from risk seeking to risk aversion, but were not monotonically related to outcome magnitude. The results contradict both Markowitz's theory, which would predict a reversal in the opposite direction, and prospect theory, which would predict global risk seeking.

\section{$<$ Insert Figure 1 about here>}

Table 1 shows the preference groups that emerged at the disaggregate level. A preference pattern with more than one preference shift is classified as 'inconsistent.' Comfortable majorities (75\% for $10 x$ choices, $60 \%$ for $2 x$ choices) changed from risk seeking to risk aversion in gains, but differed in losses. The Wavering were always the largest group. We considered how they would most likely have been classified had they not been inconsistent in losses. We did so by counting, for each participant among the Wavering, the number of matches with (1) each of the Markowitzian preference patterns (initial choices of the sure thing, subsequent choices of the gamble), (2) each of the non-Markowitzian patterns (initial choices of the gamble, subsequent choices of the sure thing), (3) the Cautious pattern (all choices of the sure thing), and (4) the Audacious pattern (all choices of the gamble), and assigning the participant to the group with the highest number of matches. Only those who matched the patterns of two or more groups equally well were not reclassified. The results are also included in Table 1.

<Insert Table 1 about here>

In $10 x$ choices, the Markowitzians represented $20 \%$ of the sample, the Audacious 3\%, and, not included in the table, other participants exhibiting prospect theory (PT) compatible preference patterns $3 \%$. The Cautious were the largest group, representing $28 \%$ of the sample. This is reflected in the aggregate data (top panel of Figure 1): Risk preferences reversed from risk seeking to risk aversion in gains but were globally risk averse in losses. In $2 x$ choices, the Markowitzians represented $7 \%$ of the sample, and those exhibiting the twofold pattern of global risk aversion in gains and global risk seeking in losses, the pattern predicted by prospect theory, $1 \%$. The non-Markowitzians were the largest group, representing $31 \%$ of the sample. This is also reflected in the aggregate data (bottom panel of Figure 1): Risk preferences reversed from risk seeking to risk aversion in both gains and losses. 
As already evident from the above summary, the composition of the preference groups changed dramatically across $10 x$ and $2 x$ choices. When going from $10 x$ to $2 x$ choices, the shares of the Markowitzians and the Cautious fell significantly while the shares of the Audacious and the nonMarkowitzians rose significantly. We will next discuss how prospect theory can cover the four groups, and explain why the shares of the respective groups rose or fell.

\section{Dispersion and Migration Effects:}

\section{A Prospect-Theoretical Interpretation}

We view the four preference groups as a behavioral reflection of four evaluation families, which we name M (after the Markowitzians), A (Audacious), N (Non-Markowitzians), and C (Cautious). The names refer to the preference pattern exhibited by the evaluation family in $2 x$ choices. The groups differ in their value function and probability-weighing function, and have the following Characteristics:

a. All groups have a value function that is decreasingly elastic in gains.

b. Families $\mathrm{M}$ and $\mathrm{A}$ have a value function that is decreasingly elastic in losses.

c. Families $\mathrm{M}$ and $\mathrm{A}$ have a value function that is more elastic in gains than in losses (gain amplification).

d. Families $\mathrm{N}$ and $\mathrm{C}$ have a value function that is increasingly elastic in losses.

e. Family $\mathrm{C}$ has the most elevated probability-weighing function, and family $\mathrm{A}$ has the least elevated probability-weighing function (see top left panel in Figure 2).

f. All four families have a more elevated probability-weighing function than in standard prospect theory, overweighing low to moderate probabilities and underweighing high ones (see top left panel in Figure 2).

While the names of the evaluation families are the initials of the preference pattern exhibited in $2 x$ choices, we will show that, in $10 x$ choices, it is possible for family A to exhibit the Markowitzian pattern, and for family $\mathrm{C}$ to exhibit the non-Markowitzian pattern. This is the migration effect, as summarized in Table 2, and as reflected in the changing composition of preference groups across Experiments 1 and 2. 
<Insert Table 2 about here>

\subsection{Gains}

We begin with the case of gains, in which all evaluation families exhibit the Markowitzian reversal from risk seeking to risk aversion. This, as discussed earlier, requires that the probabilityweighing function outweighs the value function for small outcomes, but is outweighed by it for large ones. Defining a value-ratio function as $R(x, p)=v(x) / v(x / p)$, we have $R(m x, p)>w(p)>R(x, p)$ for $m>1$ and $x>0$,

The case of gains is shown in the top right panel of Figure 2. In what we call an ' $R w$-mapping plot,' probability-weighing functions are superimposed on value-ratio functions. Two value-ratio functions, one for small $x$ and another for large $x$ (i.e., $m x$ ), are plotted as a function of $p$. Because the value function is concave over gains, both $R(x, p)$ and $R(m x, p)$ are greater than $p$. Moreover, because the value function is decreasingly elastic in gains (Characteristic $a), R(m x, p)$ is greater than $R(x, p)$. The three probabilityweighing functions from the top left panel of Figure 2, which overweigh low to moderate probabilities (Characteristic $f$ ), are superimposed on the value-ratio functions. For low to moderate probabilities, $w(p)$ is greater than $R(x, p)$ but smaller than $R(m x, p)$, yielding the Markowitzian reversal from risk seeking to risk aversion.

\subsection{Losses}

We now turn to losses, where the four evaluation families have distinct preference patterns. The $R w$-mapping plots are shown in the bottom left (families $\mathrm{M}$ and $\mathrm{A}$ ) and bottom right (families $\mathrm{N}$ and $\mathrm{C}$ ) panels of Figure 2.

\subsubsection{Markowitzians and Audacious}

We first consider families $\mathrm{M}$ and $\mathrm{A}$. Their value function is decreasingly elastic in losses (Characteristic $b$ ), so that, as in the case of gains, $R(m x, p)$ is greater than $R(x, p)$. Furthermore, their value function is less elastic in losses than in gains (gain amplification; Characteristic $c$ ), so that the value-ratio functions are more elevated in losses than in gains (compare the top right and bottom left panels of Figure 2). This has no consequences in family $\mathrm{M}$, whose probability-weighing function is more elevated than that 
of family A (Characteristic $e$ ): For low to moderate probabilities, $w(p)$ is greater than $R(x, p)$ but smaller than $R(m x, p)$, yielding the Markowitzian reversal from risk aversion to risk seeking. In family A, however, gain amplification does have consequences. For moderate probabilities, $w(p)$ is smaller than $R(x$, $p)$ as well as $R(m x, p)$, yielding the Audacious pattern of global risk seeking. For low probabilities, which are overweighed more than moderate ones, $w(p)$ is greater than $R(x, p)$ but smaller than $R(m x, p)$, yielding the Markowitzian pattern. Thus, for low probabilities, family A exhibits the Markowitzian pattern, but, for moderate probabilities, family A exhibits the Audacious pattern of global risk seeking. Because family A goes from Markowitzian to Audacious when going from $10 x$ to $2 x$ choices, but family M remains Markowitzian, we should see a fall in the share of the Markowitzians and a rise in the share of the Audacious (as we see in Table 1). This leads us to the first prediction of our prospect-theoretical analysis: Those who are Markowitzians in $10 x$ choices will either remain Markowitzians or become Audacious in $2 x$ choices. That is, when going from $10 x$ to $2 x$ choices, there should be many cases changing from Markowitzian to Audacious but few cases changing in the opposite direction.

\subsubsection{Cautious and non-Markowitzians}

For families $\mathrm{N}$ and $\mathrm{C}$, the situation is similar to that of families $\mathrm{M}$ and $\mathrm{A}$, except that they have an increasingly elastic value function over losses (Characteristic $d$ ), so that $R(x, p)$ is greater than $R(m x, p){ }^{4}$ The probability-weighing function of family $\mathrm{C}$ is more elevated than that of family $\mathrm{N}$ (Characteristic $e$ ). For low to moderate probabilities, $w(p)$ is greater than $R(x, p)$ as well as $R(m x, p)$, yielding the Cautious pattern of global risk aversion. ${ }^{5}$ The probability-weighing function of family $\mathrm{N}$ is less elevated than that of family C. For moderate probabilities, $w(p)$ is smaller than $R(x, p)$ but greater than $R(m x, p)$, yielding the non-Markowitzian reversal from risk seeking to risk aversion. For low probabilities, which are overweighed more than moderate ones, $w(p)$ is greater than $R(x, p)$ as well as $R(m x, p)$, yielding the Cautious pattern. Thus, for low probabilities, family N exhibits the Cautious pattern, but, for moderate probabilities, family $\mathrm{N}$ exhibits the non-Markowitzian pattern. Because family $\mathrm{N}$ goes from Cautious to non-Markowitzian when going from $10 x$ to $2 x$ choices, but family $\mathrm{C}$ remains Cautious, we should see a fall in the share of the Cautious and a rise in the share of the non-Markowitzians (as we see in Table 1). 
This leads us to the second prediction of our prospect-theoretical analysis: Those who are Cautious in $10 x$ choices will either remain Cautious or become Non-Markowitzians in $2 x$ choices. That is, when going from $10 x$ to $2 x$ choices, there should be many cases changing from Cautious to non-Markowitzian but few cases changing in the opposite direction.

In Experiment 3, we test the above predictions by asking the same participants to make both $10 x$ and $2 x$ choices.

\section{Experiment 3}

\section{1. Method}

\subsubsection{Participants and payment}

Population and recruitment were the same as in Experiments 1 and 2. The participants were 569 members of eLab, including 193 men and 365 women (11 participants did not fill in their gender), averaging 38 years of age. The great majority had some college or a Bachelor's degree. They were entered in a lottery offering a 1 in 50 chance to win a $\$ 50$ Amazon.com gift certificate.

\subsubsection{Stimuli and procedure}

Participants made a series of seven $10 x$ choices and a series of seven $2 x$ choices for gains and for losses. The magnitude of the sure outcome ranged from $\$ 0.25$ to $\$ 250,000$ in multiples of 10 . There were five between-participants conditions. In four conditions, the magnitude of the sure thing increased across choice tasks (as in Experiments 1 and 2). The order of $10 x$ and $2 x$ choices, and, within them, the order of gains and losses, was counterbalanced between participants. In a fifth condition, all choices were randomized. This condition was included as a check on the robustness of our results, and potential order effects (see Harrison et al., 2005). Randomization did not affect the results (see Discussion), and our analyses will therefore collapse over all five conditions.

\subsection{Results}

Figure 3 shows the aggregate results for the $10 x$ and $2 x$ choices, which replicate those from Experiments 1 and 2: A reversal from risk seeking to risk aversion in gains combined with global risk aversion (10x choices) and a reversal from risk seeking to risk aversion ( $2 x$ choices) in losses. Table 3 
shows the disaggregate results, which also replicate those from the Experiments 1 and 2. First, a majority changed from risk seeking to risk aversion in gains, but dispersed into different preference groups in losses (dispersion effect). While our non-standard modification of prospect theory accounts for a majority of the cases (62\% in the $10 x$ choices and $51 \%$ in the $2 x$ choices), standard prospect theory, augmented with decreasing elasticity, accounts for $31 \%$ of the cases in the $10 x$ choices (21\% Markowitzians, $6 \%$ Audacious, and $4 \%$ others), and only $2 \%$ of the cases in the $2 x$ choices (those exhibiting the twofold pattern of global risk aversion in gains and global risk seeking in losses). Second, when going from $10 x$ to $2 x$ choices, the shares of the Markowitzians and the Cautious fell significantly while the shares of the Audacious and the non-Markowitzian patterns rose significantly (migration effect).

<Insert Figure 3 about here>

<Insert Table 3 about here>

To test the predictions about migration between preference groups, Table 4 reports observed minus expected number of transitions between preference groups across $10 x$ and $2 x$ choices, upon reclassification of the Wavering. The predictions are confirmed. First, those who were Markowitzians in $10 x$ choices tended to either remain Markowitzians or become Audacious in $2 x$ choices (they also tended to become Wavering), whereas the Audacious tended to remain Audacious. Among those who made a transition between these two preference groups across $10 x$ and $2 x$ choices, a large majority (86\%) were Markowitzians in $10 x$ choices but Audacious in $2 x$ choices, $\chi^{2}(1)=7.14, p=.00$. Second, those who were Cautious in $10 x$ choices tended to either remain Cautious or become non-Markowitzians in $2 x$ choices, whereas non-Markowitzians tended to remain non-Markowitzians. Among those who made a transition between these two preference groups across $10 x$ and $2 x$ choices, a large majority (86\%) were Cautious in $10 x$ choices but non-Markowitzians in $2 x$ choices, $\chi^{2}(1)=15.21, p=.00$.

<Insert Table 4 about here> 


\subsection{Discussion}

The results from Experiment 3 show that we have arrived at a successful extension of prospect theory to individual differences in risky choice. This individual-difference analysis accounts for dispersion and migration effects.

Our non-standard modification of prospect theory covers a majority of participants in both $10 x$ and $2 x$ choices, whereas standard prospect theory covers only a minority, and, in $2 x$ choices, an almost nonexistent minority. Thus, to achieve a 'decent' coverage, one must abandon the view of a typical decision maker, and allow for individual differences between decision makers, which, in our prospecttheoretical interpretation of the results, are individual differences in the elasticity of the value function and the elevation of the probability-weighing function.

Our analysis covers a significantly greater percentage of participants in $10 x$ choices than in $2 x$ choices, both in the between-participants analysis (75\% in Experiment 1 vs. $60 \%$ in Experiment 2, see Table 1) and in the within-participant analysis (62\% vs. 51\% in Experiment 3, see Table 3). This may indicate that the overweighing of a .1 probability is more common than the overweighing of a .5 probability, as standard prospect theory would imply. However, only a very small percentage of participants (2\%) exhibited the preference pattern predicted by prospect theory in $2 x$ choices (the twofold pattern of global risk aversion in gains and global risk seeking in losses). It thus seems that, when going from $10 x$ to $2 x$ choices, about $10 \%$ of the participants slip through the net, and start doing things that neither standard prospect theory nor our non-standard modification of prospect theory accommodates.

As mentioned earlier, we included a randomized order condition. Randomization did not affect our conclusions about the differential composition of preference groups in $10 x$ and $2 x$ choices. To substantiate this, we computed, separately for the randomized order condition and the four nonrandomized order conditions, the difference in incidence rates of the Markowitzians, the nonMarkowitzians, the Cautious, and the Audacious between $10 x$ and $2 x$ choices. Figure 4 shows the result: Our conclusions are the same, regardless of whether choices were randomized or not. 
Anomalies to Markowitz's Hypothesis 21

\section{Are We Doing Something Wrong? \\ The Fourfold Pattern in Outcome Probability}

\subsection{Theory}

We have reported results that are surprisingly at odds with Markowitz's theory and standard prospect theory. This may raise doubts about our method. However, there seems nothing wrong with our method: We simply present participants with a series of choices between a sure thing and a gamble.

Nonetheless, we felt the need to perform a check on our method. We did so by testing a fourfold pattern of risk preferences that has proven to be very robust in past studies, and that our method should be able to reproduce.

Our focus has been on the M4 pattern, which concerns how risk preferences change as a function of outcome magnitude. This pattern differs from the fourfold pattern of risk preferences hypothesized by Kahneman and Tversky (1979; Tversky \& Kahneman, 1992), the 'KT4 pattern,' which concerns how risk preferences change as a function of outcome probability. As illustrated by Tversky and Fox (1995), and Tversky and Wakker (1995), people will prefer a 1/20 chance of gaining \$100 to gaining $\$ 5$ for sure (risk seeking), but will prefer gaining \$95 for sure to a 19/20 chance of gaining \$100 (risk aversion). Conversely, they will prefer losing $\$ 5$ for sure to a $1 / 20$ chance of losing $\$ 100$ (risk aversion), but will prefer a 19/20 chance of losing \$100 to losing \$95 for sure (risk seeking). The KT4 pattern has proven to be very robust in past studies (e.g., Cohen et al., 1987, Table 2; Fehr-Duda et al., 2010, Figure 2; Hershey \& Schoemaker, 1980, Table 3; Kahneman \& Tversky, 1979, Table 1; Tversky \& Kahneman, 1992, Table 4; but see Harbaugh et al., 2009), and our method should be able to reproduce it.

Prospect theory explains the KT4 pattern as follows. The probability-weighing function overweighs the low probability of $1 / 20$, contributing to risk seeking in gains and risk aversion in losses, but underweighs the high probability of 19/20, contributing to risk aversion in gains and risk seeking in losses. The value function is concave in gains and convex in losses, contributing to risk aversion in gains and risk seeking in losses. The KT4 pattern will occur when, for the low probability of $1 / 20$, the probability-weighing function outweighs the value function, i.e., $w(p)>v(p x) / v(x)$. 
Just as prospect theory predicts alternatives to the M4 pattern in choices between $x$ and $(10 x, .1)$, or $10 x$ choices, so does it predict alternatives to the KT4 pattern in choices between $p x$ and $(x, p)$, or ' $p$ choices.' Prospect theory predicts the KT4 pattern when the probability-weighing function outweighs the value function for low probabilities. However, this does not necessarily happen. When the probabilityweighing function is outweighed by the value function for both gains and losses, prospect theory predicts a twofold pattern of global risk aversion in gains and global risk seeking in losses. Under symmetric elasticity, and in the absence of domain-specific probability weighing, this is the only possible alternative to the KT4 pattern, because, if the probability-weighing function does not outweigh the value function in gains, it does not outweigh the value function in losses either, i.e., $v(p x) / v(x)=v(-p x) / v(-x)>w(p)$ for $x$ $>0$.

Under loss amplification, the probability-weighing function may outweigh the value function for losses but not for gains, i.e., $v(p x) / v(x)>w(p)>v(-p x) / v(-x)$, producing a threefold pattern of global risk aversion in gains and a reversal from risk aversion to risk seeking in losses. Conversely, under gain amplification, the probability-weighing function may outweigh the value function for gains but not for losses, i.e., $v(-p x) / v(-x)>w(p)>v(p x) / v(x)$, producing a threefold pattern of global risk seeking in losses and a reversal from risk seeking to risk aversion in gains.

In sum, prospect theory predicts four preference patterns, one of which is the KT4 pattern. Markowitz's theory does not predict the KT4 pattern, because, in his theory, the probability-weighing function is an identity function, i.e., $w(p)=p$.

\subsection{Stimuli and procedure}

In Experiments 2 and 3, the participants first made 2 choices between $p x$ and $(x, p)$, or $p$ choices, for gains and for losses, before proceeding to the Markowitzian choice series. The magnitude of the risky outcome was $\$ 100$, and its probability was .05 and .95 . In Experiment 2, the probability of the risky outcome increased across choice tasks, and the order of gains and losses was counterbalanced between participants. In Experiment 3, there were three between-participants conditions. In two conditions, the probability of the risky outcome increased across choice tasks, and the order of gains and losses was 
counterbalanced between participants (as in Experiment 2). In a third condition, all choices were randomized. An example of a gain question, as presented to the participants, is the choice between "a $19 / 20$ chance of receiving $\$ 100$ " and “definitely receiving $\$ 95$," and an example of a loss question is the choice between "a $1 / 20$ possibility of having to pay $\$ 100$ " and "definitely having to pay $\$ 5$."

\subsection{Results}

Figure 5 shows the aggregate results for Experiments 2 and 3. There is strong evidence for the KT4 pattern: A reversal from risk seeking to risk aversion in gains and from risk aversion to risk seeking in losses. The disaggregate results were also very similar for the two experiments, as summarized below.

$<$ Insert Figure 5 about here>

\subsubsection{Experiment 2}

A majority (55\%) exhibited the four PT compatible preference patterns. Three of these groups contributed to the KT4 pattern at the aggregate level: Those exhibiting the KT4 pattern, i.e., a reversal from risk seeking to risk aversion in gains and from risk aversion to risk seeking in losses (28\%), those exhibiting a reversal in losses but global risk aversion in gains (16\%), and those exhibiting a reversal in gains but global risk seeking in losses $(6 \%)$. The fourth group, those exhibiting global risk aversion in gains and global risk seeking in losses, represented $5 \%$ of the sample.

Confirmatory cases in $2 x$ choices tended to be confirmatory cases in $p$ choices. A majority (61\%) of those who, in $2 x$ choices, changed from risk seeking to risk aversion in gains exhibited, in $p$ choices, a PT compatible preference pattern, $\chi^{2}(1)=7.51, p=.01$, and the incidence rate of PT compatible preference patterns was significantly lower (47\%) among those who, in $2 x$ choices, did not change from risk seeking to risk aversion in gains, $\chi^{2}(1)=5.19, p=.02$.

\subsubsection{Experiment 3}

A majority (51\%) exhibited the four PT compatible preference patterns. Three of these groups contributed to the KT4 pattern at the aggregate level: Those exhibiting the KT4 pattern (26\%), those exhibiting a reversal in losses but not in gains (14\%), and those exhibiting a reversal in gains but not in losses (6\%). The fourth group, those exhibiting no reversal, represented $6 \%$ of the sample. 
Confirmatory cases in $10 x$ and $2 x$ choices tended to be confirmatory cases in $p$ choices. On the one hand, a majority (60\%) of those who, in $10 x$ choices, changed from risk seeking to risk aversion in gains exhibited, in $p$ choices, a PT compatible preference pattern, $\chi^{2}(1)=14.81, p=.00$, and the incidence rate of PT compatible preference patterns was significantly lower (37\%) among those who, in $10 x$ choices, did not change from risk seeking to risk aversion in gains, $\chi^{2}(1)=28.14, p=.00$. On the other hand, a majority (58\%) of those who, in $2 x$ choices, changed from risk seeking to risk aversion in gains exhibited, in $p$ choices, a PT compatible preference pattern, $\chi^{2}(1)=7.64, p=.01$, and the incidence rate of PT compatible preference patterns was significantly lower (45\%) among those who, in $2 x$ choices, did not change from risk seeking to risk aversion in gains, $\chi^{2}(1)=5.19, p=.02$.

\subsection{Conclusion}

Our method not only reproduces the KT4 pattern at the aggregate level, but also shows how this pattern emerges from four different preference groups at the disaggregate level. In addition, those cases who confirmed standard (and non-standard) prospect theory's predictions for $p$ choices tended to be cases who confirmed non-standard prospect theory's predictions for $10 x$ and $2 x$ choices.

\section{General Discussion}

Harry Markowitz hypothesized a fourfold pattern of risk preferences, the 'M4 pattern,' with risk aversion for large gains and small losses, but risk seeking for small gains and large losses. Daniel Kahneman and Amos Tversky later hypothesized another fourfold pattern of risk preference, the 'KT4 pattern,' with risk aversion for likely gains and unlikely losses, but risk seeking for unlikely gains and likely losses. To explain the M4 pattern, Markowitz preserved the expectation principle, i.e., $w(p)=p$, but replaced a concave utility function over final states of wealth by a triply inflected value function over changes of wealth, which is concave over large gains and small losses, but convex over small gains and large losses. To explain the KT4 pattern, Tversky and Kahneman replaced the expectation principle by probability weighing, with an overweighing of low probabilities, i.e., $w(p)>p$, and an underweighing of moderate to high ones, i.e., $w(p)<p$. Because Tversky and Kahneman also replaced the triply inflected 
value function by a singly inflected value function, which is concave over gains and convex over losses, prospect theory can explain the M4 pattern only by combining the overweighing of low probabilities with a value function that is decreasingly elastic over gains and losses of increasing magnitude.

We confirmed the KT4 pattern, which was already well-established, but not the M4 pattern. While Markowitz's hypothesis is correct for gains, it is generally wrong for losses. In reaction to this evidence, one might propose to preserve the expectation principle, and endow each preference group with its appropriately inflected value function. This proposal, however, does not explain why the composition of preference groups changes across probability levels. Nor does it explain the KT4 pattern. To explain this, we need probability weighing, and, therefore prospect theory.

We have shown that prospect theory can accommodate our evidence if we make non-standard assumptions about both the value function and the probability-weighing function. These assumptions however, do not change the central tenets of prospect theory. For instance, there is nothing in prospect theory that prohibits an overweighing of moderate probabilities, or a non-constant elasticity of the value function. Moreover, experimental arrangements and individual differences may affect the functional forms of prospect theory, which have, indeed, shown great variability across studies, and across individuals within any particular study (e.g., Gonzalez \& Wu, 1999). More generally, the stability of functional forms in decision making has been called into question (e.g., Ungemach et al., 2011). One variable aspect of prospect theory is probability weighing. Our experimental arrangement, involving Markowitzian choice series, suggests that moderate probabilities are overweighted, whereas other experimental arrangements suggest that they are underweighted (e.g., Abdellaoui, 2000, Figure 5; Bleichrodt \& Pinto, 2000, Figure 2, Tversky \& Fox, 1995, Figure 9; Tversky \& Kahneman, 1992, Figure 1). Substantive theory should explain this variability.

Markowitz framed his theory as an explanation of why people simultaneously purchase insurance and gambles. Upon identifying implausible implications of the analysis offered by Friedman and Savage (1948), Markowitz proposed his triply inflected value function over changes of wealth, in which a small loss (the price of an insurance or a gamble) is barely different from the status quo, whereas the large 
potential calamity against which one insures and the large potential prize for which one gambles are not. Our analysis shows that Markowitz's proposal is not viable either. So we must sketch the implications of our analysis for the behavior that he set out to explain.

In standard prospect theory, the purchase of insurance and gambles is promoted by the overweighing of low probabilities (Kahneman \& Tversky, 1979). The willingness to purchase insurance is mitigated by the convexity of the value function over losses, and the willingness to purchase gambles is mitigated both by the concavity of the value function over gains and by loss aversion when the loss (the price of the gamble) is segregated from the potential gain (the prize; see Kahneman \& Tversky, 1979). Apart from these general principles, however, there are, in our non-standard modification of prospect theory, particularities of different groups in the evaluation of risky prospects.

We identified four groups, each with their unique combination of a value function and a probability-weighing function. Let us name them by the preferences they expressed in the 50/50 gambles involving losses. Using the same functional forms as in Figure 2, the reservation prices for insuring against a .01 probability of losing $\$ 1,000$ (fair price is $\$ 10$ ) are:

\$16 (Markowitzians),

$\$ 11$ (Audacious),

\$193 (non-Markowitzians), and

$\$ 255$ (Cautious).

Three relations can be established. First, the Markowitzians and the Audacious react in the same way to losses, but the Markowitzians put a greater weight on the .01 probability of losing than the Audacious, so that their reservation price is higher. Second, the Markowitzians and the non-Markowitzians put the same weight on the .01 probability of losing, but the non-Markowitzians, with their increasingly elastic value function over losses, react more strongly to the $\$ 1,000$ loss than the Markowitzians, with their decreasingly elastic value function over losses, so that their reservation price is higher. Third, the nonMarkowitzians and the Cautious react in the same way to losses, but the Cautious put a greater weight on the .01 probability of losing than the non-Markowitzians, so that their reservation price is higher. 
Altogether, we have a differentiated market for insurance, with the willingness to participate depending on probability weighing and outcome valuation. In realistic situations, with companies selling insurance well above the fair price, the Markowitzians and the Audacious would, in this simulation, probably not participate, whereas the non-Markowitzians and the Cautious probably would. This is good news for the insurance companies, because the non-Markowitzians and the Cautious were relatively large groups in our experiments.

In making the transition from insurance to gambles, note that those who are 'Audacious' in losses are, because they put less weight on risky outcomes, more cautious than Markowitzians in gains, and that those who are 'Cautious' in losses are, because they put more weight on risky outcomes, more audacious than non-Markowitzians in gains. Using the same functional forms as before, and assuming a lossaversion coefficient $\lambda$ equal to 2 , the reservation prices for a gamble offering a .01 probability of winning $\$ 1,000$ are:

\$38 (Markowitzians),

\$23 (Audacious, now more cautious),

\$15 (non-Markowitzians), and

\$21 (Cautious, now more audacious).

Three observations can be made. First, the non-Markowitzians put a higher price on the insurance than the Markowitzians, but put a lower price on the gamble, and for the same reason. These two groups react in the same way to gains, and put the same weight on the .01 probability of winning, but the nonMarkowitzians, with their increasingly elastic value function over losses, react more strongly to the price of the gamble than the Markowitzians, with their decreasingly elastic value function over losses, so that their reservation price is lower. Second, the Markowitzians and the 'Audacious' put a higher price on the gamble than on the insurance, which means that, in this simulation, gain amplification (a stronger reaction to the potential prize than to the potential calamity) outweighs loss aversion (a disproportionately stronger reaction to the price of the gamble than to the potential prize). Third, the non-Markowitzians and the 'Cautious' put a significantly higher price on the insurance than on the gamble. This has two reasons. One 
is that their value function is increasingly elastic in losses but decreasingly elastic in gains (a stronger reaction to the potential calamity than to the potential prize). The other reason is loss aversion (a disproportionately stronger reaction to the price of the gamble than to the potential prize). The combined influence of these two factors yields, in these groups, the pronounced discrepancy between willingness to insure and willingness to gamble. Altogether, our analysis implies not only inter-individual differences, but also pronounced intra-individual differences in the reaction to insurance and gambles.

Kahneman and Tversky (1979) recognized that the purchase of insurance and gambles can be determined by many factors, and that the psychophysics of risk and value is only one of them. The same caveat applies to our analysis. Moreover, the above analysis of insurance and gambles does not cover all realistic situations of decision under risk. If insuring is a form of 'playing safe,' so is 'safe sex,' in which a relatively small inconvenience (a condom) averts a large potential cost (premature death). Similarly, if gambling is a form of 'taking a chance,' so is 'unsafe sex,' in which a relatively small enjoyment invites a large potential cost. The latter example is an interesting one, because, in gambling, incurring a small loss opens the possibility of obtaining a large gain, whereas, in unsafe sex, obtaining a small gain opens the possibility of incurring a large loss. Alternative forms of playing safe and taking a chance may receive closer attention in future analyses.

Our modification of prospect theory essentially involves assumptions about the elevation of the probability-weighing function (depending on the person, it is more or less elevated), and assumptions about the elasticity of the value function (depending on the person and the domain, its elasticity decreases or increases with outcome magnitude). Stake-dependent elasticity has not previously been associated with prospect theory. After Tversky and Kahneman (1992), users of prospect theory routinely resort to a power value function, the elasticity of which is constant in outcome magnitude. We found decreasing elasticity in gains. In losses, however, some seem to combine diminishing sensitivity with decreasing elasticity, while others seem to combine it with increasing elasticity. Thus, decreasing elasticity is not a universal property of the value function over losses, but constant elasticity is certainly disconfirmed by our findings. 
Finally, our modification of prospect theory does not address a more fundamental limitation of prospect theory, which is that it cannot account for a greater preference uncertainty in losses than in gains. In what we reported, this is indicated by people whose preferences do not conform to a definite pattern in losses (the Wavering), but we also observed systematically longer choice times for losses than for gains, especially among the Wavering. One suggestion would be to incorporate the functional forms of prospect theory into decision field theory (Busemeyer \& Townsend, 1993), where 'attention weights' are given by prospect theory's probability-weighing function and 'valences' are given by prospect theory's value function. The key contribution of decision field theory would be a steeper 'goal gradient' for losses than for gains, as reflected in longer choice times and greater choice inconsistency. Apart from that, the qualitative predictions of prospect theory, on which we focused in this paper, would be preserved.

In sum, we examined Markowitz's hypothesis about decision under risk, and, instead of the hypothesized pattern, we obtained dispersion and migration effects. Combined, these two effects show that loss aversion does not fully capture the asymmetry between gains and losses. Losses not only loom larger than gains, they also engender (1) a greater diversity, with different people exhibiting different preference patterns (preference heterogeneity) and many people exhibiting preferences that do not conform to a definite pattern (preference uncertainty), and (2) a greater variability of preference patterns across risk levels. Although our analysis was restricted to the Markowitzian choice series, it covered three central issues in decision under risk: How risk preferences depend on the magnitude and sign of the outcomes, and on the level of risk involved. Markowitzian choice series consist of elementary choices between a sure thing and a gamble, and, even in this severely restricted situation, the typical decision maker does not seem to exist. The prevailing approach of modeling a typical decision maker should therefore be reconsidered. 


\section{References}

Abdellaoui, M., 2000. Parameter-free elicitation of utility and probability weighing functions. Management Science 46, 1497-1512.

Abdellaoui, M., Barrios, C., Wakker, P. P., 2007. Reconciling introspective utility with revealed preference: Experimental arguments based on prospect theory. Journal of Econometrics 138, 356-378. al-Nowaihi, A., Dhami, S., 2009. A value function that explains magnitude and sign effects. Economics Letters 105, 224-229.

Bernoulli, D., 1738. Specimen theoriae novae de mensura sortis. Commentarii academiae scientiarum imperialis petropolitanae. Translated, Bernoulli, D., 1954. Exposition of a new theory on the measurement of risk. Econometrica 22, 23-36.

Bleichrodt, H., Pinto, J. L., 2000. A parameter-free elicitation of the probability weighting function in medical decision analysis. Management Science 46, 1485-1496.

Brandstätter, E., Gigerenzer, G., Hertwig, R., 2006. The priority heuristic: Making choices without tradeoffs. Psychological Review 113, 409-432.

Busemeyer, J. R., Townsend, J. T., 1993. Decision field theory: A dynamic-cognitive approach to decision-making in an uncertain environment. Psychological Review 100, 432-459.

Cohen, M., Jaffray, J.-Y., Said, T., 1987. Experimental comparison of individual berhavior under risk and under uncertainty for gains and for losses. Organizational Behavior and Human Decision Processes $39,1-22$.

Fehr-Duda, H., Bruhin, A., Epper, T., Schubert, R., 2010. Rationality on the rise: Why relative risk aversion increases with stake size. Journal of Risk and Uncertainty 40, 147-180.

Friedman, M., Savage, L. J., 1948. The utility analysis of choices involving risk. Journal of Political Economy $56,279-304$.

Gonzalez, R., Wu, G., 1999. On the shape of the probability weighing function. Cognitive Psychology 38, 129-166. 
Green, L., Myerson, J., Ostaszewski, P., 1999. Amount of reward has opposite effects in the discounting of delayed and probabilistic outcomes. Journal of Experimental Psychology: Learning, Memory, and Cognition 25, 418-127.

Harbaugh, W. T., Krause, K., Vesterlund, L., 2009. The fourfold pattern of risk attitudes in choice and pricing tasks. Economic Journal 120, 595-611.

Harrison, G. W., Johnson, E., McInnes, M. M., Rutström, E. E., 2005. Temporal stability of estimates of risk aversion. Applied Financial Economics Letters 1, 31-35.

Hershey, J. C., Schoemaker, P. J. H., 1980. Prospect theory's reflection hypothesis: A critical examination. Organizational Behavior and Human Performance 25, 395-418.

Hogarth, R. M., Einhorn, H. J., 1990. Venture theory: A model of decision weights. Management Science $36,780-803$.

Holt, C. A., Laury, S. K., 2002. Risk aversion and incentive effects. American Economic Review 92, 1644-1655.

Kahneman, D., Tversky, A., 1979. Prospect theory: An analysis of decision under risk. Econometrica 47, 363-391.

Köbberling, V., Wakker, P. P., 2005. An index of loss aversion. Journal of Economic Theory 122, 119131.

Lichtenstein, S., Slovic, P., 1971. Reversals of preference between bids and choices in gambling decisions. Journal of Experimental Psychology 89, 46-55.

Loewenstein, G., Prelec, D., 1992. Anomalies in intertemporal choice: Evidence and an interpretation. Quarterly Journal of Economics 107, 573-597.

Loomes, G., 2010. Modeling choice and valuation in decision experiments. Psychological Review 117, 902-924.

Markowitz, H., 1952. The utility of wealth. Journal of Political Economy 60, 151-158.

Prelec, D., Loewenstein, G., 1991. Decision making over time and under uncertainty: A common approach. Management Science 37, 770-786. 
Schneider, S. L., 1992. Framing and conflict: Aspiration level contingency, the status quo, and current theories of risky choice. Journal of Experimental Psychology: Learning, Memory, and Cognition 18, 1040-1057.

Schoemaker, P. J. H., 1990. Are risk-attitudes related across domains and response modes? Management Science 36, 1451-1463.

Scholten, M., Read, D., 2010. The psychology of intertemporal tradeoffs. Psychological Review 117, 925944.

Tversky, A., Fox, C. R., 1995. Weighing risk and uncertainty. Psychological Review 102, 269-283.

Tversky, A., Kahneman, D., 1991. Loss aversion in riskless choice: A reference-dependent model. Quarterly Journal of Economics 107, 1039-1061.

Tversky, A., Kahneman, D., 1992. Advances in prospect theory: Cumulative representation of uncertainty. Journal of Risk and Uncertainty 5, 297-323.

Tversky, A., Wakker, P., 1995. Risk attitudes and decision weights. Econometrica, 63 1255-1280.

Ungemach, C., Stewart, N., Reimers, S., 2011. How incidental values from the environment affect decisions about money, risk, and delay. Psychological Science 22, 253-260.

Wakker, P. P., Köbberling, V., Schwieren, C., 2007. Prospect theory’s diminishing sensitivity versus economics' intrinsic utility of money: How the introduction of the euro can be used to disentangle the two empirically. Theory and Decision 63, 205-231.

Weber, B. J., Chapman, G. B., 2005. Playing for peanuts: Why is risk seeking more common for lowstakes gambles? Organizational Behavior and Human Decision Processes 97, 31-46. 


\section{Appendix}

Following Loewenstein and Prelec (1992), elasticity is defined as $\varepsilon_{v}(x) \equiv \partial \log (v(x)) / \partial \log (\mathrm{x})=$ $x v^{\prime}(x) / v(x)$. Thus, elasticity is the rate at which outcome value $v(x)$ changes relative to the rate at which outcome $x$ changes. Diminishing sensitivity, which is commonly stated as $v^{\prime \prime}(x)<0$ for $x>0$ and $v^{\prime \prime}(x)>0$ for $x<0$, can also be stated as $v(0)=0$ and $0<\varepsilon_{v}(x)<1$. This means, for instance, that a $10 \%$ change in the magnitude of $x$ corresponds to less than a 10\% change in the magnitude of $v(x)$.

Elasticity can be constant for all $x$, or it can vary with $x$. A power value function $v(x)=x^{\alpha}$ has constant elasticity, because $x \alpha x^{\alpha-1} / x^{\alpha}=\alpha$. The value ratio $R(x, p)=v(x) / v(x / p)$, or the ratio between the value of the sure thing and the value of the risky outcome, is inversely related to $\alpha$. When $\varepsilon_{v}(x)=\alpha=1$, we have a linear value function $v(x)=x$, and a linear value-ratio function $R(x, p)=p$. Furthermore, when 0 $<\varepsilon_{v}(x)=\alpha<1$, we have a concave value function $v(x)=x^{\alpha}$, and a concave value-ratio function $R(x, p)=$ $p^{\alpha}$. Finally, when $\varepsilon_{v}(x)=\alpha=0$, we have a constant value function $v(x)=1$, and a constant value-ratio function $R(x, p)=1$.

An example of a value function with increasing elasticity is an additive combination of two power value functions, one characterized by a lower elasticity, or more strongly diminishing sensitivity, than the other, i.e., $v(x)=x^{\alpha}+\mu x^{1-\alpha}$, where $0<\alpha<1 / 2$ and $\mu>0$. The elasticity of this value function is $\varepsilon_{v}(x)=\frac{\alpha x^{\alpha}+(1-\alpha) \mu x^{1-\alpha}}{x^{\alpha}+\mu x^{1-\alpha}}$,

which approaches $\alpha$ as $x \rightarrow 0$, and 1- $\alpha$ as $x \rightarrow \infty$. Intuitively, when $x$ is very small, most of the curvature is determined by the first term of the value function, $x^{\alpha}$, with elasticity $\alpha$. As $x$ becomes larger and larger, however, most of the value, and, therefore, most of the curvature, is determined by the second term of the value function, $x^{1-\alpha}$, with elasticity $1-\alpha$.

Diminishing sensitivity is easier to combine with decreasing elasticity than with increasing elasticity (see Scholten and Read 2010). For instance, the value function $v(x)=\log (x)$ has as its elasticity $\varepsilon_{v}(x)=1 / \log (x)$, which is clearly decreasing in $x$. A log function like this one was proposed by Bernoulli 
(1738/1954) to describe utility from final states of wealth. However, it has several shortcomings when used to describe value from changes in wealth relative to a neutral reference point. An alternative log function, proposed by Scholten and Read (2010) in the domain of intertemporal choice, is $v(x)=(1 / \alpha)$ $\log (1+\alpha x)$. The elasticity of this value function is

$\varepsilon_{v}(x)=\frac{\alpha x}{(1+\alpha x) \log (1+\alpha x)}$,

which approaches 1 as $x \rightarrow 0$, and 0 as $x \rightarrow \infty$. 


\section{Authors' Note}

Marc Scholten, associate professor with habilitation at the ISPA University Institute, Lisbon, Portugal. Daniel Read, professor of behavioral science at Warwick Business School, University of Warwick, Coventry, United Kingdom. We acknowledge the financial support received from the Fundação para a Ciência e Tecnologia (FCT), program POCI 2010, and project PTDC/PSI-PCO/101447/2008. This paper has greatly benefited from the comment given by Peter Wakker and Jerome Busemeyer. We are also very grateful to Andrew Meyer for his assistance in conducting the experiments.

Correspondence concerning this paper should be addressed to Marc Scholten, ISPA University Institute, Rua Jardim do Tabaco 34, 1149-041 Lisboa, Portugal. E-Mail: scholten@ ispa.pt. 
Anomalies to Markowitz's Hypothesis 36

TABLE 1

Experiments 1 and 2: Relative size (\%) of preference groups in choices between $x$ and $(10 x, .1)$ and choices between $x$ and $(2 x, .5)$, and difference of proportions tests with separate variance estimates. ${ }^{a}$

\begin{tabular}{|c|c|c|c|c|c|c|}
\hline \multirow[b]{2}{*}{ Designation } & \multicolumn{2}{|c|}{ Preference pattern } & \multirow[b]{2}{*}{$x$ vs. $(10 x, .1)$} & \multirow[b]{2}{*}{$x$ vs. $(2 x, .5)$} & \multirow[b]{2}{*}{$t$} & \multirow[b]{2}{*}{$p$} \\
\hline & Gains & Losses & & & & \\
\hline \multirow[t]{2}{*}{ Markowitzians } & $\mathrm{RS}-\mathrm{RA}$ & $\mathrm{RA}-\mathrm{RS}$ & 20 & 7 & -3.65 & .00 \\
\hline & & & (12) & (4) & $(-3.27)$ & $(.00)$ \\
\hline \multirow[t]{2}{*}{ Audacious } & $\mathrm{RS}-\mathrm{RA}$ & $\mathrm{RS}$ & 4 & 8 & 1.91 & .06 \\
\hline & & & (3) & (4) & $(0.97)$ & $(.33)$ \\
\hline \multirow[t]{2}{*}{ Non-Markowitzians } & $\mathrm{RS}-\mathrm{RA}$ & $\mathrm{RS}-\mathrm{RA}$ & 16 & 31 & 3.78 & .00 \\
\hline & & & (12) & (22) & $(2.86)$ & $(.00)$ \\
\hline \multirow[t]{2}{*}{ Cautious } & $\mathrm{RS}-\mathrm{RA}$ & RA & 28 & 7 & -5.76 & .00 \\
\hline & & & (26) & (6) & $(-5.57)$ & $(.00)$ \\
\hline \multirow[t]{2}{*}{ Wavering } & $\mathrm{RS}-\mathrm{RA}$ & Inconsistent & 7 & 7 & -0.30 & .76 \\
\hline & & & (23) & $(25)$ & $(0.48)$ & $(.63)$ \\
\hline Miscellaneous & & & 25 & 40 & 3.36 & .00 \\
\hline
\end{tabular}

${ }^{a}$ Numbers in parentheses are those before reclassification of the Wavering. 
TABLE 2

Summary of the migration effect.

\begin{tabular}{cll}
\hline & \multicolumn{2}{c}{ Preference group } \\
\cline { 2 - 3 } Evaluation family & \multicolumn{1}{c}{$10 x$ choices } & \multicolumn{1}{c}{$2 x$ choices } \\
\hline $\mathbf{M}$ & Markowitzians & Markowitzians \\
$\mathbf{A}$ & Markowitzians & Audacious \\
$\mathbf{N}$ & Cautious & Non-Markowitzians \\
$\mathbf{C}$ & Cautious & Cautious \\
\hline
\end{tabular}


TABLE 3

Experiment 3: Relative size (\%) of preference groups in choices between $x$ and $(10 x, .1)$ and choices between $x$ and $(2 x, .5)$, and McNemar tests. ${ }^{a}$

\begin{tabular}{|c|c|c|c|c|c|c|}
\hline \multirow[b]{2}{*}{ Designation } & \multicolumn{2}{|c|}{ Preference pattern } & \multirow[b]{2}{*}{$x$ vs. $(10 x, .1)$} & \multirow[b]{2}{*}{$x$ vs. $(2 x, .5)$} & \multirow[b]{2}{*}{$\chi^{2}$} & \multirow[b]{2}{*}{$p$} \\
\hline & Gains & Losses & & & & \\
\hline \multirow[t]{2}{*}{ Markowitzians } & $\mathrm{RS}-\mathrm{RA}$ & $\mathrm{RA}-\mathrm{RS}$ & 21 & 5 & 62.04 & .00 \\
\hline & & & (13) & (2) & $(42.96)$ & $(.00)$ \\
\hline \multirow[t]{2}{*}{ Audacious } & $\mathrm{RS}-\mathrm{RA}$ & $\mathrm{RS}$ & 6 & 9 & 4.66 & .03 \\
\hline & & & (4) & (6) & $(2.75)$ & $(.10)$ \\
\hline \multirow[t]{2}{*}{ Non-Markowitzians } & $\mathrm{RS}-\mathrm{RA}$ & $\mathrm{RS}-\mathrm{RA}$ & 16 & 25 & 19.06 & .00 \\
\hline & & & (9) & (18) & $(25.25)$ & $(.00)$ \\
\hline \multirow[t]{2}{*}{ Cautious } & $\mathrm{RS}-\mathrm{RA}$ & RA & 12 & 6 & 17.28 & .00 \\
\hline & & & (10) & (5) & (13.14) & $(.00)$ \\
\hline \multirow[t]{2}{*}{ Wavering } & $\mathrm{RS}-\mathrm{RA}$ & Inconsistent & 7 & 6 & 0.13 & .72 \\
\hline & & & $(25)$ & (19) & $(7.01)$ & $(.01)$ \\
\hline Miscellaneous & & & 38 & 49 & 19.67 & .00 \\
\hline
\end{tabular}

${ }^{a}$ Numbers in parentheses are those before reclassification of the Wavering. 
Anomalies to Markowitz's Hypothesis 39

TABLE 4

Experiment 3: Observed minus expected number of transitions between preference groups across choices between $x$ and (10x, .1) and choices between $x$ and $(2 x, .5)$, upon reclassification of the Wavering. ${ }^{a}$

\begin{tabular}{lcccccc}
\hline & \multicolumn{5}{c}{ Preference group in $2 x$ choices } \\
\cline { 2 - 7 } Preference group in 10x choices & Markowitzians & Audacious & Non-Markowitzians & Cautious & Wavering & Miscellaneous \\
\hline Markowitzians & $\mathbf{6 . 7 8}$ & $\mathbf{1 . 6 3}$ & -3.03 & -2.84 & 8.53 & -11.07 \\
Audacious & 0.31 & $\mathbf{7 . 1 9}$ & 0.13 & -0.86 & -2.02 & -4.75 \\
Non-Markowitzians & -4.75 & 0.09 & $\mathbf{2 9 . 8 6}$ & -1.22 & -2.69 & -21.29 \\
Cautious & -1.69 & -4.15 & $\mathbf{7 . 7 8}$ & $\mathbf{9 . 9 4}$ & -0.43 & -11.45 \\
Wavering & 0.89 & 2.49 & -2.84 & 0.68 & 0.47 & -1.68 \\
Miscellaneous & -1.55 & -7.24 & -31.88 & -5.70 & -3.86 & 50.23 \\
\hline
\end{tabular}

${ }^{a} \chi^{2}(25)=187.58, p=.00$. 


\section{Figure Captions}

Figure 1. Top panel: Aggregate results from Experiment $1(N=189)$, showing the probability of choosing sure thing $x$ over the gamble $(10 x, .1)$ as a function of outcome magnitude and sign. Bottom panel: Aggregate results from Experiment $2(N=255)$, showing the probability of choosing sure thing $x$ over the gamble $(2 x, .5)$ as a function of outcome magnitude and sign. The whiskers are $95 \%$ confidence intervals. Figure 2. A prospect-theoretical interpretation of dispersion and migration effects. The top left panel shows the probability-weighing functions of family $\mathrm{C}$ (solid curve), families $\mathrm{M}$ and $\mathrm{N}$ (dashed curve), and family A (dotted curve). The other three panels show $R w$-mapping plots, in which probability-weighing functions are superimposed on value-ratio functions. The top right panel shows the case of gains for all evaluation families. The bottom left panel shows the case of losses for families $\mathrm{M}$ and $\mathrm{A}$, while the bottom right panel shows the case of losses for families $\mathrm{N}$ and $\mathrm{C}$.

Figure 3. Aggregate results from Experiment $3(N=569)$ : On the left, the probability of choosing sure thing $x$ over the gamble $(10 x, .1)$, and, on the right, the probability of choosing sure thing $x$ over the gamble $(2 x, .5)$, as a function of outcome magnitude and sign.

Figure 4. Experiment 3: Difference $(d)$ in incidence rates of the Markowitzians, the non-Markowitzians, the Cautious, and the Audacious between $2 x$ and $10 x$ choices, separately for the randomized order condition and the non-randomized order conditions.

Figure 5. Aggregate results from Experiment 2 (top panel) and Experiment 3 (bottom panel): The probability of choosing sure thing $p x$ over the gamble $(x, p)$ as a function of outcome probability and sign. 
FIG. 1
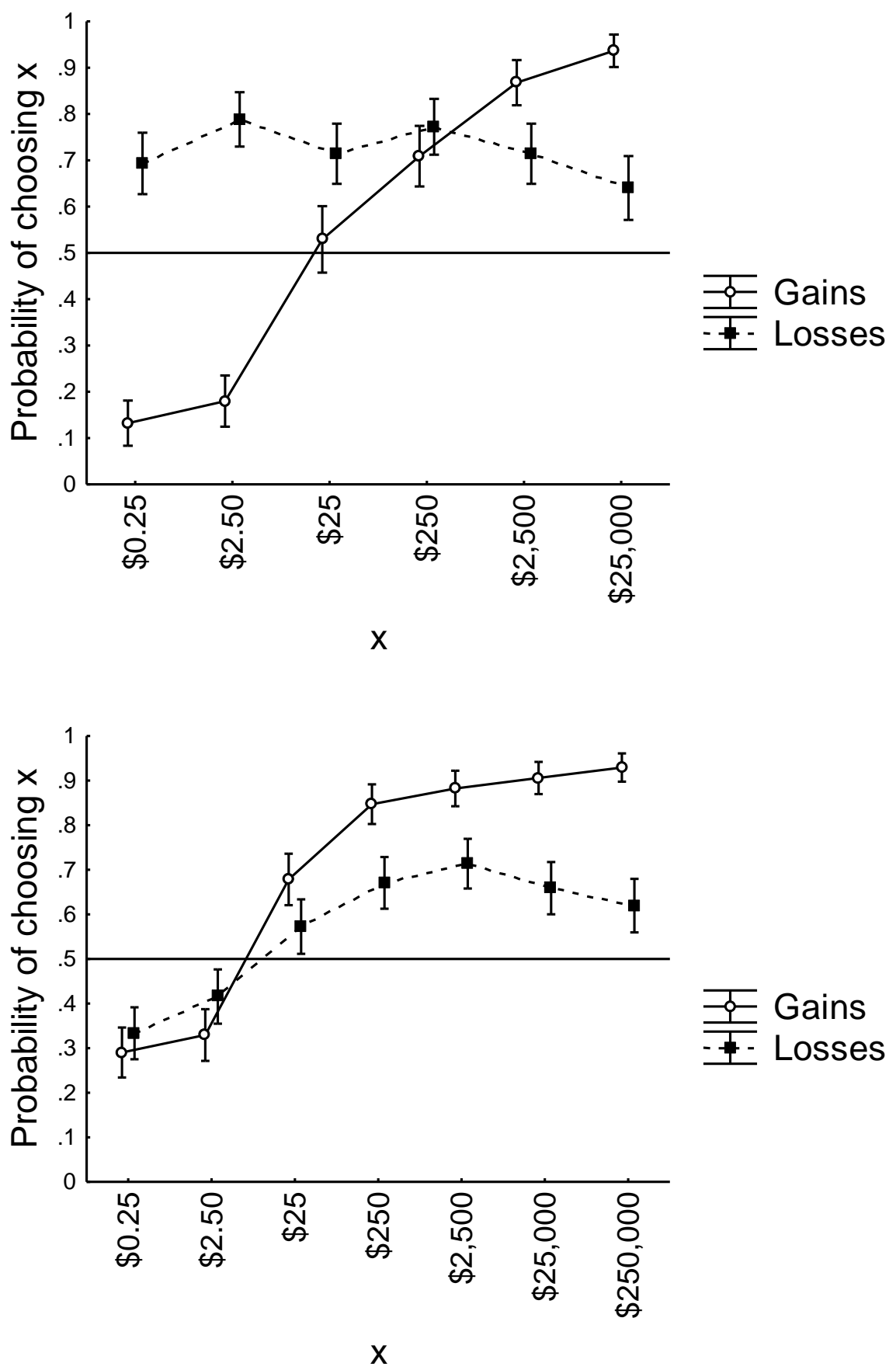
Anomalies to Markowitz's Hypothesis 42

FIG.2
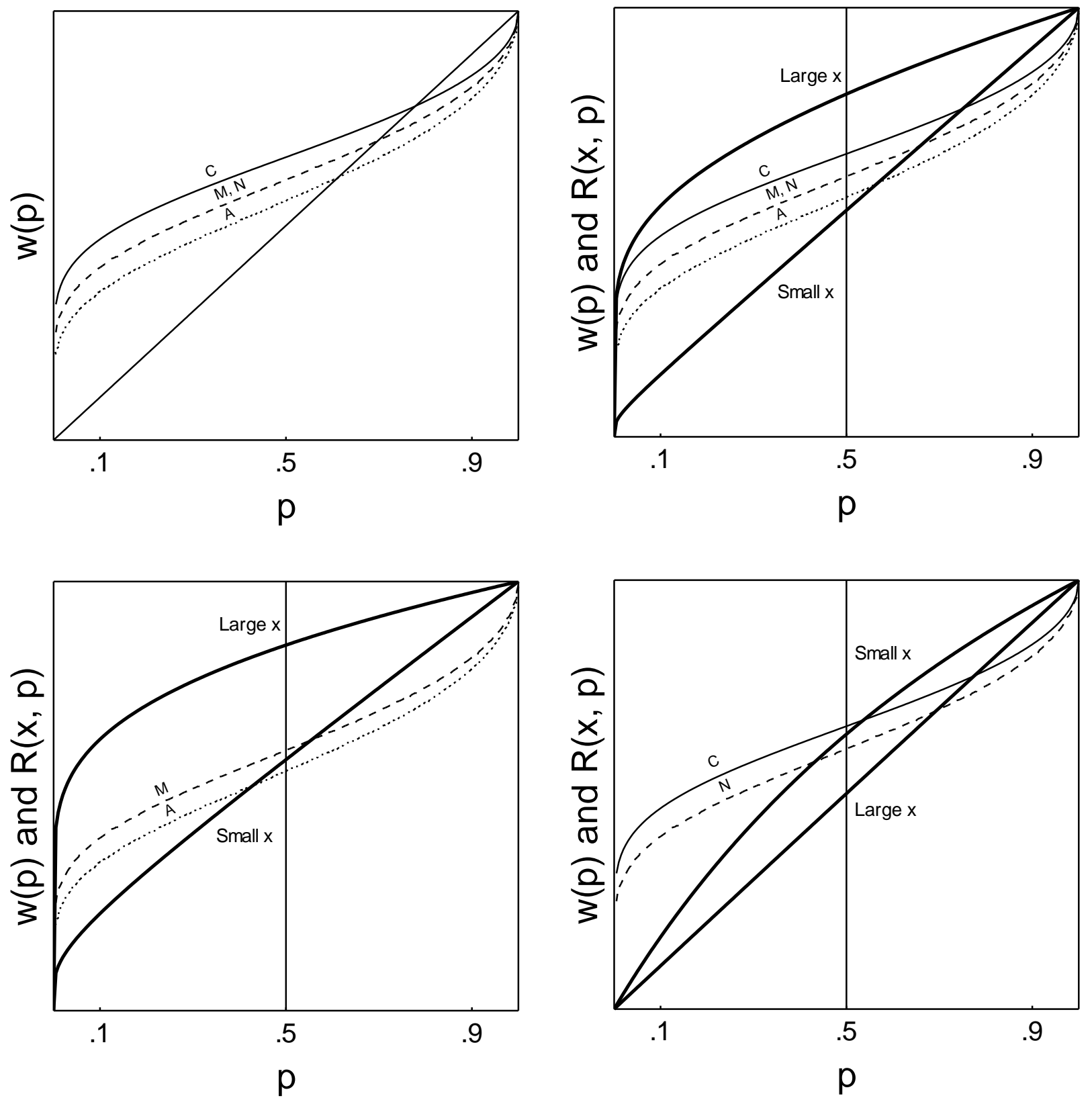
FIG. 3
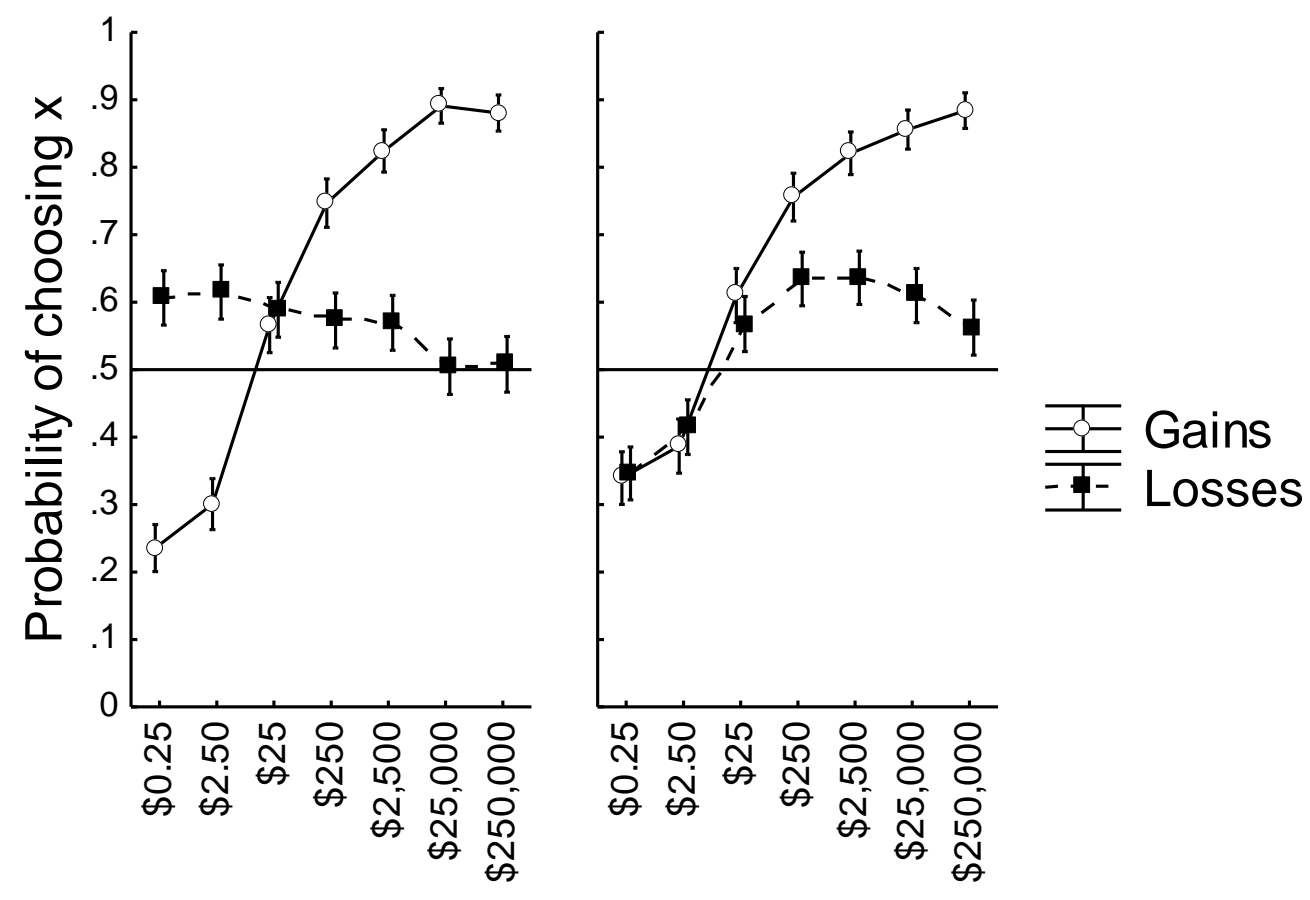

10x choices

$2 x$ choices 
FIG. 4

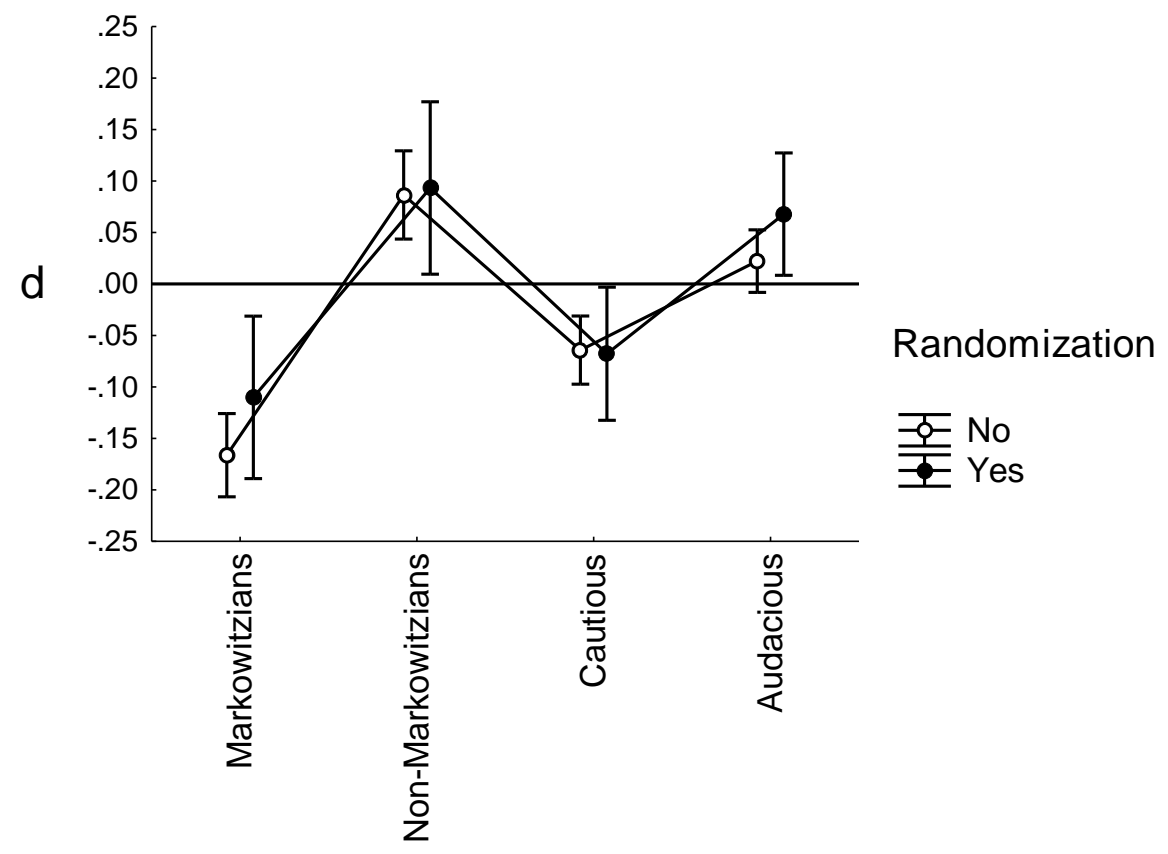


Anomalies to Markowitz's Hypothesis 45

FIG. 5
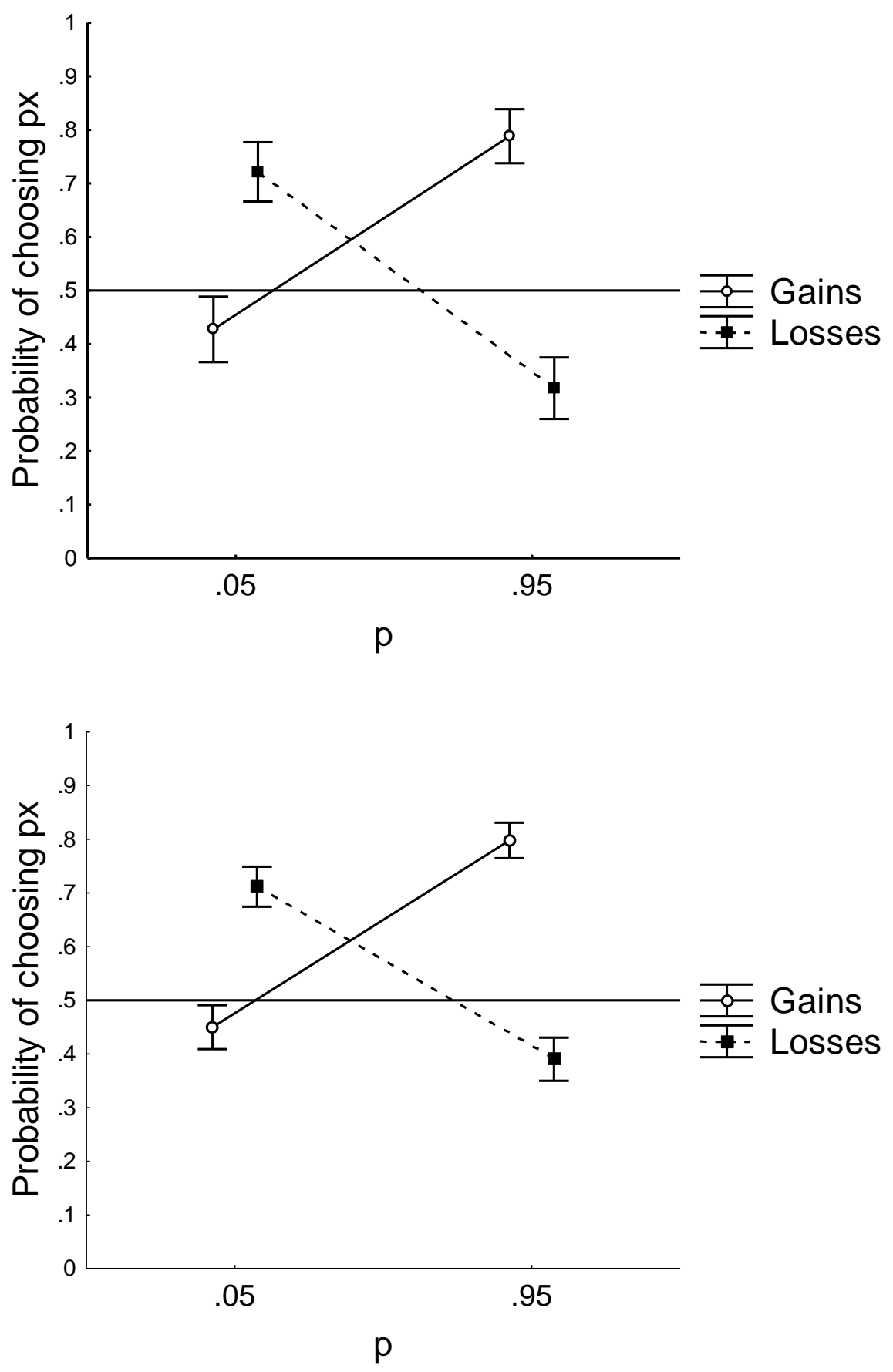


\section{Footnotes}

${ }^{1}$ We use the term 'preference reversal' to denote a reversal of risk preferences as revealed by a person's choices. This should be distinguished from the 'preference reversals' between choice and pricing discovered by Lichtenstein and Slovic (1971), in which a person chooses a safer gamble but assigns a higher cash equivalent to a riskier one.

${ }^{2}$ Another possibility is that the probability-weighing function always outweighs the value function: $w(1 / 10)>\frac{v(m x)}{v(10 m x)}>\frac{v(x)}{v(10 x)}$.

This, however, is not a realistic possibility in prospect theory. The probability weight $w(1 / 10)$ generally remains far removed from its upper limit of 1 , but, given a decreasingly elastic value function $v$, the value ratio $v(x) / v(10 x)$ moves toward 1 as the magnitude of $x$ increases. It will thus become increasingly unlikely that $w(1 / 10)$ exceeds $v(x) / v(10 x)$.

${ }^{3}$ Weber and Chapman (2005, Table 5) report results that are consistent with those of Hershey and Schoemaker's (1980) study. However, they did not examine choices between a sure thing and a gamble, but rather choices between two gambles. In their study, aggregate choices were globally and increasingly risk averse in gains and globally and increasingly risk seeking in losses. That is, there were no reversals, but larger gains resulted in more risk aversion and larger losses resulted in more risk seeking. As in Hershey and Schoemaker's (1980) study, choice probabilities were closer to chance level for losses than for gains.

${ }^{4}$ Comparison of the bottom panels in Figure 2 shows that $R(x, p)$ is less elevated for small $x$ in families $\mathrm{N}$ and $\mathrm{C}$ than for large $x$ in families $\mathrm{M}$ and $\mathrm{A}$, meaning that $v$ is more elastic in the former case than in the latter. We do not include this among the Characteristics, because value functions differ not only in whether elasticity increases or decreases, but also in the range within which, and the rate at which, it increases or decreases. For instance, in the Appendix, the elasticity of the increasingly elastic value 
function ranges from $\alpha$ to $1-\alpha$, whereas the elasticity of the decreasingly elastic value function ranges from 1 to 0 . Therefore, elasticity lies in a higher and narrower range in the former case than in the latter. ${ }^{5}$ A situation where the probability-weighing function always outweighs the value function is a realistic possibility under increasing elasticity, not under decreasing elasticity (see Footnote 2). 